\title{
ON GENERALIZED HARMONIC ANALYSIS
}

\author{
BY
}

\author{
KA-SING LAU ${ }^{1}$ AND JONATHAN K. LEE ${ }^{2}$
}

\begin{abstract}
Motivated by Wiener's work on generalized harmonic analysis, we consider the Marcinkiewicz space $\mathfrak{T}^{p}(\mathbf{R})$ of functions of bounded upper average $p$ power and the space $\mathcal{T}^{p}(\mathbf{R})$ of functions of bounded upper $\boldsymbol{p}$ variation. By identifying functions whose difference has norm zero, we show that $\mathfrak{T}^{p}(\mathbf{R}), 1<p$ $<\infty$, is a Banach space. The proof depends on the result that each equivalence class in $\Upsilon^{p}(\mathbf{R})$ contains a representative in $L^{p}(\mathbf{R})$. This result, in turn, is based on Masani's work on helixes in Banach spaces.

Wiener defined an integrated Fourier transformation and proved that this transformation is an isometry from the nonlinear subspace $\mathcal{W}^{2}(\mathbf{R})$ of $\mathscr{T}^{2}(\mathbf{R})$ consisting of functions of bounded average quadratic power, into the nonlinear subspace $Q^{2}(\mathbf{R})$ of ${ }^{2}(\mathbf{R})$ consisting of functions of bounded quadratic variation. By using two generalized Tauberian theorems, we prove that Wiener's transformation $W$ is actually an isomorphism from $\Re^{2}(\mathbf{R})$ onto $\mathcal{V}^{2}(\mathbf{R})$. We also show by counterexamples that $W$ is not an isometry on the closed subspace generated by थ $(\mathbf{R})$.
\end{abstract}

1. Introduction. The purpose of this paper is to find out how Wiener's generalized harmonic analysis [18] fits into the framework of contemporary functional analysis.

For a complex valued Borel measurable function $f$ on $\mathbf{R}$ such that $\lim _{T \rightarrow \infty}(2 T)^{-1} \int_{-T}^{T}|f(x)|^{2} d x$ exists, Wiener [18] defined the integrated Fourier transformation $g=W(f)$ of $f$ as

$$
g(u)=\frac{1}{2 \pi}\left(\int_{-\infty}^{-1}+\int_{1}^{\infty} f(x) \frac{e^{-i u x}}{-i x} d x+\int_{-1}^{1} f(x) \frac{e^{-i u x}-1}{-i x} d x\right) .
$$

We call $W$ the Wiener transformation. By using a deep Tauberian theorem, he then proved that the mean square modulus of the above function $f$ equals the quadratic variation of its transformation $g$, i.e.

$$
\lim _{T \rightarrow \infty} \frac{1}{2 T} \int_{-T}^{T}|f(x)|^{2} d x=\lim _{h \rightarrow 0^{+}} \frac{1}{2 h} \int_{-\infty}^{\infty}|g(u+h)-g(u-h)|^{2} d u .
$$

Now, for all $f \in L_{\text {loc }}^{2}(\mathbf{R})$, let

$$
\|f\|=\|f\|_{\Re^{2}}=\varlimsup_{T \rightarrow \infty}\left(\frac{1}{2 T} \int_{-T}^{T}|f(x)|^{2} d x\right)^{1 / 2}
$$

Received by the editors November 10, 1978.

AMS (MOS) subject classifications (1970). Primary 42A68; Secondary 42A32, 46B99.

Key words and phrases. Banach spaces, generalized harmonic analysis, helixes, Marcinkiewicz spaces, Tauberian theorem, upper $p$-variation, Wiener transformation.

${ }^{1}$ Partially supported by the Faculty of Arts and Science research grant of the University of Pittsburgh.

${ }^{2}$ Partially supported by NSF Grants GP7808 and GP7809. 
and

$$
\mathfrak{T}^{2}(\mathbf{R})=\left\{f: f \in L_{\text {loc }}^{2}(\mathbf{R}),\|f\|<\infty\right\} .
$$

Note that the set of functions $\mathcal{W}^{2}(\mathbf{R})$ for which the limit on the left-hand side of (1.1) exists and is finite is a nonlinear subspace of the linear space $\Re^{2}(R)$.

Next, for all Borel measurable $g$ on $\mathbf{R}$, let

$$
\|g\|=\|g\|_{\gamma^{2}}=\varlimsup_{h \rightarrow 0^{+}}\left(\frac{1}{2 h} \int_{-\infty}^{\infty}|g(u+h)-g(u-h)|^{2} d u\right)^{1 / 2}
$$

and

$$
\mathcal{V}^{2}(\mathbf{R})=\{g: g \text { is Borel measurable and }\|g\|<\infty\} .
$$

Then the set of functions for which the limit on the right-hand side of (1.1) exists and is finite is a nonlinear subspace of the linear space $\mathcal{V}^{2}(\mathbf{R})$.

Similarly, we can define the classes of functions $\mathfrak{N}^{p}(\mathbf{R})$ and $\mathfrak{W}^{p}(\mathbf{R})$. Both $\mathfrak{N}^{p}(\mathbf{R})$ and $\mathcal{V}^{p}(\mathbf{R})$ are normed linear spaces when two functions in any one of the spaces whose differences have norm zero are identified. Marcinkiewicz [13] and independently Bohr and Følner [3] showed that $\mathscr{T}^{p}(\mathbf{R})$ is complete, but the question of the completeness of $\mathcal{T}^{p}(\mathbf{R})$ has been open.

In $\S 3$, we show that $\mathcal{T}^{p}(\mathbf{R})$ is complete for $1<p<\infty$. For this, we find that all usual methods of proving completeness (cf. e.g. [5], [12]) fail. We have to appeal to the theory of helixes in a Banach space $X$, i.e. continuous functions $x_{(\cdot)}$ on $\mathbf{R}$ to $X$ such that for all $a, b, t \in \mathbf{R}, U_{t}\left\{x_{b}-x_{a}\right\}=x_{b+t}-x_{a+t}$, where $\left\{U_{t}\right\}_{t \in \mathbf{R}}$ is a strongly continuous group of isometries [8], [14]. Using results from the theory of helixes, we are able to show that each equivalence class in $\Upsilon^{p}(\mathbf{R}), 1<p<\infty$, contains a function in $L^{p}(\mathbf{R})$. This enables us to get hold of a limit for any Cauchy sequence in $\mathcal{V}^{p}(\mathbf{R}), 1<p<\infty$.

The case $p=1$ has been considered by Nelson recently [17]; he showed that $\mathcal{V}^{1}(\mathbf{R})$ is isometric isomorphic to the space of countably additive, Borel measures on $\mathbf{R}$ with finite variation. Hence $\mathfrak{V}(\mathbf{R})$ is also complete.

Equation (1.1) shows that the Wiener transformation $W$ is an isometry on the nonlinear subspace $\mathscr{W}(\mathbf{R})$ of $\mathscr{T}^{2}(\mathbf{R})$. In $\S 5$, we show that $W$ is an isomorphism from $\Re^{2}(\mathbf{R})$ onto $\Upsilon^{2}(\mathbf{R})$ with

$$
\|W\|=\left(\int_{0}^{\infty} \tilde{h}(x) d x\right)^{1 / 2} \text { and }\left\|W^{-1}\right\|=\left(\max _{x>0} x h(x)\right)^{-1 / 2}
$$

where $h(x)=\left(2 \sin ^{2} x\right) / \pi x^{2}, x \geqslant 0$, and $\tilde{h}(x)=\sup _{t>x} h(t), x>0$ (i.e., $\tilde{h}$ is the smallest decreasing function which dominates $h$ ). The proof depends on two special types of Tauberian theorems which we will develop in $\$ 4$ (Theorems 4.5 and 4.6).

It follows from (1.4) that

$$
\|W\|=\left(\int_{0}^{\infty} \tilde{h}(x) d x\right)^{1 / 2}>\left(\int_{0}^{\infty} \frac{2 \sin ^{2} x}{\pi x^{2}} d x\right)^{1 / 2}=1
$$


and

$$
\left\|W^{-1}\right\|=\left(\max _{x>0} \frac{2 \sin ^{2} x}{\pi x}\right)^{-1 / 2}>1 .
$$

(Numerically, $\|W\| \approx 1.05$ and $\left\|W^{-1}\right\| \approx 1.49$.) In view of (1.1), it is natural to ask whether $W$ restricted on $\left\langle\mho^{2}(\mathbf{R})\right\rangle$, the closed linear subspace generated by $\mho^{2}(\mathbf{R})$, is an isometry. We answer this question negatively and thereby disprove a conjecture by Masani [16].

Finally, we observe that the Wiener transformation $W$ is also a bounded linear operator from $\mathfrak{T}^{p}(\mathbf{R})$ into $\mathfrak{V}^{p^{\prime}}(\mathbf{R}), 1<p<2,1 / p+1 / p^{\prime}=1$.

ACKNOWLEDGEMENT. The proof of the completeness of the space $\Upsilon^{p}(\mathbf{R})$ and of the fact that $W$ is an isomorphism on $\mathscr{T}^{2}(\mathbf{R})$ onto $\mathcal{T}^{2}(\mathbf{R})$, the definition of $\tilde{h}$ and the proof of the result

$$
\|W\|<\left(\int_{0}^{\infty} \tilde{h}(x) d x\right)^{1 / 2} \text { and }\left\|W^{-1}\right\|<\left(\max _{x>0} x h(x)\right)^{-1 / 2}
$$

were given by Lee in his Indiana University doctoral dissertation in 1971 (unpublished) (cf. [10], [11]). The equality (1.4) and the Tauberian results are due to Lau. Both authors would like to express their gratitude to Professor Masani for his supervision and comments on this work. Their thanks are also due to the referee for many helpful suggestions in simplifying the paper.

2. The space $\mathfrak{T}^{p}(\mathbf{R})$. Throughout, we assume that $f$ is a complex valued, Borel measurable function on $\mathbf{R}$. Let $w$ be a positive Borel measurable function. We will use $L^{p}(\mathbf{R}, w(x) d x)$ to denote the Banach space of functions $f$ such that

$$
\|f\|=\left(\int_{-\infty}^{\infty}|f(x)|^{p} w(x) d x\right)^{1 / p}<\infty
$$

Let $M^{p}(\mathbf{R}), 1<p<\infty$, denote the set of locally $p$-integrable functions $f$ such that

$$
\|f\|=\sup _{1<T<\infty}\left(\frac{1}{2 T} \int_{-T}^{T}|f(x)|^{p} d x\right)^{1 / p}<\infty .
$$

Let $I^{p}(\mathbf{R})$ be the subspace of $f$ in $M^{p}(\mathbf{R})$ such that

$$
\varlimsup_{T \rightarrow \infty} \frac{1}{2 T} \int_{-T}^{T}|f(x)|^{p} d x=0 .
$$

Let $\mathfrak{T}^{p}(\mathbf{R})$ be the Marcinkiewicz space defined as in the introduction and let $\Psi^{p}(\mathbf{R})$ be the set of $f$ in $\mathscr{N}^{p}(\mathbf{R})$ such that

$$
\lim _{T \rightarrow \infty} \frac{1}{2 T} \int_{-T}^{T}|f(x)|^{p} d x
$$

exists.

Proposition 2.1. Let $1 \leqslant p<\infty$ and let $a>0$. Then

$$
M^{p}(\mathbf{R}) \varsubsetneqq L^{p}\left(\mathbf{R}, \frac{d x}{1+|x|^{1+a}}\right) .
$$


Proof. For any $T>1$,

$$
\begin{aligned}
\int_{-T}^{T} \frac{|f(x)|^{p}}{1+|x|^{1+a}} d x= & \int_{-T}^{T} \frac{1}{1+|x|^{1+a}} d\left(\int_{0}^{x}|f(y)|^{p} d y\right) \\
= & \frac{1}{1+T^{1+a}} \int_{-T}^{T}|f(y)|^{p} d y \\
& +(1+a) \int_{0}^{T} \frac{x^{a}}{\left(1+x^{1+a}\right)^{2}}\left(\int_{-x}^{x}|f(y)|^{p} d y\right) d x \\
\leqslant & \frac{2 T}{1+T^{1+a}} \frac{1}{2 T} \int_{-T}^{T}|f(y)|^{p} d y \\
& +2(1+a) \int_{0}^{T} \frac{1}{1+x^{1+a}}\left(\frac{1}{2 x} \int_{-x}^{x}|f(y)|^{p} d y\right) d x
\end{aligned}
$$

This implies

$$
\int_{-\infty}^{\infty} \frac{|f(x)|^{p}}{1+|x|^{1+a}} d x \leqslant k\left(\|f\|_{M^{p}}\right)^{p}
$$

where

$$
k=2(1+a) \int_{0}^{\infty} \frac{d x}{1+x^{1+a}}
$$

Hence

$$
M^{p}(\mathbf{R}) \subseteq L^{p}\left(\mathbf{R}, \frac{1}{1+|x|^{1+a}}\right)
$$

The strict inclusion follows from the fact that $f(x)=(\log x)^{1 / p} \chi_{[1, \infty)}(x)$ is in $L^{p}\left(\mathbf{R}, 1 /\left(1+|x|^{1+a}\right)\right)$ but is not in $M^{p}(\mathbf{R})$.

Proposition 2.2. Let $1 \leqslant p<\infty$. Then

(i) $M^{p}(\mathbf{R})$ is a Banach space,

(ii) $\mathfrak{T}^{p}(\mathbf{R})$ is isometric isomorphic to the quotient space $M^{p}(\mathbf{R}) / I^{p}(\mathbf{R})$ under the natural identification.

Proof. We leave the simple proof of (i) to the reader. To prove (ii), we identify functions in $\Re^{p}(\mathbf{R})$ whose differences have zero norm. We will still use $f$ to denote the equivalence class of $f$ in $\mathfrak{T}^{p}(\mathbf{R})$. The map $\tau: M^{p}(\mathbf{R}) \rightarrow \mathscr{K}^{p}(\mathbf{R})$ with $\tau(f)=f$ is clearly a contraction. It is also a surjection, for if $f$ is in $\mathfrak{T}^{p}(\mathbf{R})$, we let

$$
f^{\prime}(x)= \begin{cases}f(x), & |x|>1 \\ 0, & |x|<1\end{cases}
$$

Then $f^{\prime} \in M^{p}(\mathbf{R})$ and $\left\|f-f^{\prime}\right\|_{\mathscr{T} P}=0$. Hence $\tau\left(f^{\prime}\right)=f^{\prime}=f$ in $\mathscr{T}^{p}(\mathbf{R})$. Also note that $\tau^{-1}(0)=I^{p}(\mathbf{R})$. This induces a bijection $\bar{\tau}: M^{p}(\mathbf{R}) / I^{p}(\mathbf{R}) \rightarrow \mathscr{N}^{p}(\mathbf{R})$ and $\|\bar{\tau}\|$ $<1$. To show that $\bar{\tau}$ is an isometry, we need only show that

$$
\inf _{g \in I^{p}(\mathbf{R})}\|f+g\|_{M^{p}}<\varlimsup_{T \rightarrow \infty}\left(\frac{1}{2 T} \int_{-T}^{T}|f(x)|^{p} d x\right)^{1 / p} .
$$


But this follows directly from

$$
\begin{aligned}
& \sup _{1<T}\left(\frac{1}{2 T} \int_{-T}^{T}\left|f(x)-f \chi_{[-a, a]}(x)\right|^{p} d x\right) \\
& \quad<\sup _{a<T}\left(\frac{1}{2 T} \int_{-T}^{T}|f(x)|^{p} d x\right) \quad \forall a>1
\end{aligned}
$$

In the following, we will give an example that $\mho^{p}(\mathbf{R})$ is not a linear subspace in $\Re^{p}(\mathbf{R})$ : Let $E_{n}=[(2 n) !,(2 n+1) !], n>0$, and let $f=\chi_{\cup_{n} E_{n}}$,

$$
g(x)=\left\{\begin{array}{ll}
\chi_{\cup_{n} E_{n}}(x)-\frac{1}{2}, & x>0, \\
0, & x<0,
\end{array} \text { and } h(x)= \begin{cases}\frac{1}{2}, & x>0, \\
0, & x<0 .\end{cases}\right.
$$

It is clear that $f=g+h$,

$$
\lim _{T \rightarrow \infty} \frac{1}{2 T} \int_{-T}^{T}|g|^{p} d x=\lim _{T \rightarrow \infty} \frac{1}{2 T} \int_{0}^{T}\left(\frac{1}{2}\right)^{p} d x=\left(\frac{1}{2}\right)^{p+1}
$$

and

$$
\lim _{T \rightarrow \infty} \frac{1}{2 T} \int_{-T}^{T}|h|^{p} d x=\left(\frac{1}{2}\right)^{p+1} .
$$

Hence $g, h \in \mathcal{Q}^{p}(\mathbf{R})$. Observe also that

$$
\begin{aligned}
\lim _{n \rightarrow \infty} \frac{1}{2(2 n) !} \int_{-(2 n) !}^{(2 n) !}|f|^{p} & =\lim _{n \rightarrow \infty} \frac{1}{2(2 n) !} \int_{0}^{(2 n-1) !}|f|^{p} \\
& \leqslant \lim _{n \rightarrow \infty} \frac{(2 n-1) !}{2(2 n) !}=0
\end{aligned}
$$

and

$$
\begin{aligned}
\lim _{n \rightarrow \infty} \frac{1}{2(2 n+1) !} \int_{-(2 n+1) !}^{(2 n+1) !}|f|^{p} & =\lim _{n \rightarrow \infty} \frac{1}{2(2 n+1) !} \int_{0}^{(2 n+1) !}|f|^{p} \\
& >\lim _{n \rightarrow \infty} \frac{(2 n+1) !-(2 n) !}{2(2 n+1) !}=\frac{1}{2}
\end{aligned}
$$

This shows that $f \notin \mathcal{W}^{p}(\mathbf{R})$ and $\mathcal{W}^{p}(\mathbf{R})$ is not a linear subspace of $\mathfrak{T}^{p}(\mathbf{R})$.

We remark that in [9], we prove that for $1<p<\infty$, each $f \in \mathcal{Q S}^{p}(\mathbf{R})$ with $\|f\|=1$ is an extreme point of the unit sphere $S\left(\Re^{p}(\mathbf{R})\right)$. The set of such $f$, however, does not exhaust all extreme points of $S\left(\Re^{p}(\mathbf{R})\right)$. For $p=1, S\left(\mathscr{N}^{1}(\mathbf{R})\right)$ does not contain any extreme points. The nonlinear subspace $\mathcal{W}^{p}(\mathbf{R})$ has also been studied by Masani in [15] where he introduced vector graph theory and conditional Banach spaces. For other properties of $\mathscr{T}^{p}(\mathbf{R})$ and its subspaces, the reader may refer to [1], [3] and [9].

3. The spaces $\mathcal{V}^{p}(\mathbf{R})$ and their completeness. For each $h \in \mathbf{R}$, we define $\tau_{h}$ and $\Delta_{h}$ as

$$
\left(\tau_{h} f\right)(x)=f(x+h) \text { and } \Delta_{h} f=\left(\tau_{h}-1\right) f
$$

where $x \in \mathbf{R}$ and $f$ is a Borel measurable function on $\mathbf{R}$. Let $\mathfrak{V}^{p}(\mathbf{R})$ be defined as in the introduction; it follows directly from the definition that for $f \in \mathcal{T}^{p}(\mathbf{R})$, 


$$
\begin{aligned}
\|f\| & =\varlimsup_{h \rightarrow 0^{+}}\left(\frac{1}{2 h} \int_{-\infty}^{\infty}\left|\left(\tau_{h}-\tau_{-h}\right) f(x)\right|^{p} d x\right)^{1 / p} \\
& =\varlimsup_{h \rightarrow 0^{+}}\left(\frac{1}{h} \int_{-\infty}^{\infty}\left|\left(\tau_{h}-1\right) f(x)\right|^{p} d x\right)^{1 / p} .
\end{aligned}
$$

By identifying functions whose differences have zero norm, it is easy to prove that $\left(\Upsilon^{p}(\mathbf{R}),\|\cdot\|\right)$ is a normed linear space. In the following, we will show that each $f \in \mathfrak{P}^{p}(\mathbf{R}), 1<p<\infty$, is equivalent to a $g \in L^{p}(\mathbf{R})$, i.e., $\|f-g\|_{\uparrow p}=0$. This fact will be useful in proving the completeness of $\mathfrak{W}^{p}(\mathbf{R}), 1<p<\infty$ (Theorem 3.6) and the surjectivity of the Wiener transformation from $\mathfrak{T}^{2}(\mathbf{R})$ onto $\mathfrak{V}^{2}(\mathbf{R})$ (Theorem 5.2).

Let $A$ be a subset in a Banach space $X$; we will use $\langle A\rangle$ to denote the closed linear subspace generated by $A$. Let $x_{(\cdot)}$ be a continuous function on $\mathbf{R}$ to $X$; we call $\delta_{x}=\left\langle\left\{x_{b}-x_{a}: a, b \in \mathbf{R}\right\}\right\rangle$ the chordal subspace of the curve $x_{(\cdot)}$. The function $x_{(\cdot)}$ is a helix in $X$ if there exists a strongly continuous group of isometries $\left\{U_{t}\right\}_{t \in \mathbf{R}}$ on $\mathcal{S}_{x}$ onto $\mathcal{S}_{x}$ such that, for any $t$ in $\mathbf{R}$,

$$
U_{t}\left(x_{b}-x_{a}\right)=x_{b+t}-x_{a+t}
$$

$\left\{U_{t}\right\}_{t \in \mathbf{R}}$ is called the shift group of the helix $x_{(\cdot)}$.

Theorem 3.1 (MASANi [14]). Let $x_{(\cdot)}$ be a helix in $X$ with shift group $\left\{U_{t}\right\}_{t \in \mathbf{R}}$ Then

$$
\alpha_{x}=\int_{0}^{\infty} e^{-t}\left(x_{0}-x_{t}\right) d t
$$

(Bochner integral) exists and is in $\mathfrak{\complement}_{x}$. Moreover,

$$
x_{b}-x_{a}=\left(U_{b}-U_{a}-\int_{a}^{b} U_{t} d t\right)\left(\alpha_{x}\right) \quad \forall a, b \in \mathbf{R} .
$$

We call $\alpha_{x}$ the average vector of the helix $x_{(\cdot)}$.

LEMMA 3.2. Let $1<p<\infty$ and let $f \in \mathcal{V}^{p}(\mathbf{R})$. If $x_{t}=\tau_{t} f-f$, then $x_{(\cdot)}$ is a helix in $L^{p}(\mathbf{R})$ with shift group $\left\{\tau_{t}\right\}_{t \in \mathbf{R}}$ and the average vector is given by

$$
\int_{0}^{\infty} e^{-t}\left(f-\tau_{t} f\right) d t \in \delta_{x} \subseteq L^{p}(\mathbf{R})
$$

PRoof. Since $f \in \mathcal{V}^{p}(\mathbf{R}), x_{t}=\left(\tau_{t}-1\right) f \in L^{p}(\mathbf{R})$ and

$$
\lim _{h \rightarrow 0^{+}} \int_{-\infty}^{\infty}\left|\left(\tau_{h}-1\right) f(\lambda)\right|^{p} d \lambda=0 .
$$

It follows that for any $t \in \mathbf{R}$,

$$
\begin{aligned}
\lim _{h \rightarrow 0^{+}} \int_{-\infty}^{\infty}\left|x_{t+h}(\lambda)-x_{t}(\lambda)\right|^{p} d \lambda & =\lim _{h \rightarrow 0^{+}} \int_{-\infty}^{\infty}\left|\left(\tau_{t+h}-\tau_{t}\right) f(\lambda)\right|^{p} d \lambda \\
& =\lim _{h \rightarrow 0^{+}} \int_{-\infty}^{\infty}\left|\left(\tau_{h}-1\right) f(\lambda)\right|^{p} d \lambda=0
\end{aligned}
$$


Hence $x_{(\cdot)}: \mathbf{R} \rightarrow L^{p}(\mathbf{R})$ is continuous. By definition, we can show that, for any $a, b$ and $t \in \mathbf{R}, \tau_{t}\left(x_{b}-x_{a}\right)=x_{b+t}-x_{a+t}$. This implies that $\left\{\tau_{t}\right\}_{t \in \mathbf{R}}$ is a strongly continuous group of isometries from $\delta_{x}\left(\subseteq L^{p}(\mathbf{R})\right)$ onto $\delta_{x}$ and $x_{(\cdot)}$ is a helix in $L^{p}(\mathbf{R})$ with shift group $\left\{\tau_{t}\right\}_{t \in \mathbf{R}}$. Finally, by Theorem 3.1, there is a $g \in \delta_{x} \subseteq L^{p}(\mathbf{R})$ such that

$$
g=\int_{0}^{\infty} e^{-t}\left(x_{0}-x_{t}\right) d t=\int_{0}^{\infty} e^{-t}\left(f-\tau_{t} f\right) d t
$$

Theorem 3.3. Let $f \in \mathfrak{P}^{p}(\mathbf{R}), 1<p<\infty$. Then there exists a $g \in L^{p}(\mathbf{R})$ such that $\|f-g\|_{\uparrow p}=0$.

Proof. Let $g=\int_{0}^{\infty} e^{-t}\left(x_{0}-x_{t}\right) d t$ be as in Lemma 3.2. For any $h>0$, we have (Theorem 3.1)

$$
\left(\tau_{h}-\tau_{-h}\right)(g-f)=\int_{-h}^{h} \tau_{t} g d t
$$

and ([8, p. 82])

$$
\left\|\int_{-h}^{h} \tau_{t} g d t\right\|_{p}<\int_{-h}^{h}\left\|\tau_{t} g\right\|_{p} d t<2 h\|g\|_{p}
$$

Hence

$$
\|f-g\|_{\mathfrak{N}^{p}}=\varlimsup_{h \rightarrow 0^{+}}\left(\frac{1}{2 h}\right)^{1 / p}\left\|\left(\tau_{h}-\tau_{-h}\right)(g-f)\right\|_{p}=0 .
$$

The theorem is not true for $p=1$ as $\mathfrak{V}(\mathbf{R})$ is isometric isomorphic to the space of countably additive Borel measures on $\mathbf{R}$ with finite variation [17].

In the following, we will consider the completeness of $\mathfrak{T}^{p}(\mathbf{R}), 1<p<\infty$. The case $p=1$ follows directly from the above isometric characterization of $\mathcal{W}(\mathbf{R})$.

Let $B\left(L^{p}(\mathbf{R})\right)$ denote the space of bounded linear operators on $L^{p}(\mathbf{R})$. For any $a, n$ in $\mathbf{R}$ with $a<b$, we let

$$
I_{a, b}=\frac{1}{b-a} \int_{a}^{b} \tau_{t} d t
$$

the integral being a Riemann integral in the strong operator topology in $B\left(L^{p}(\mathbf{R})\right)$ ([8, pp. 62-67]). We note the following facts:

$$
\begin{gathered}
\forall s \in \mathbf{R}, \quad \tau_{s} I_{a, b}=I_{a, b} \tau_{s}, \\
\forall f \in L^{p}(\mathbf{R}), \quad \lim _{h \rightarrow 0^{+}} I_{0, h}(f)=f \text { in } L^{p}(\mathbf{R}), \\
\forall h>0, \quad\left\|I_{0, h}\right\|<1, \\
\forall a, b \in \mathbf{R}, a<b \quad \text { and } \forall f \in L^{p}(\mathbf{R}), \quad I_{a, b}(f) \in \mathscr{D}_{A},
\end{gathered}
$$

where $\mathscr{D}_{A}$ is the domain of the infinitesimal generator $A$ of the translation group $\left\{\tau_{h}\right\}_{h \in \mathbf{R}}$ on $L^{p}(\mathbf{R})([8$, p. 307]). It is well known that $A$ is the restriction of the differential operator on $\mathscr{D}_{A}$ and $\mathscr{D}_{A}=\left\{f \in L^{p}(\mathbf{R}): f\right.$ is absolutely continuous and $\left.f^{\prime} \in L^{p}(\mathbf{R})\right\}$. Let $g \in \mathscr{D}_{A}$; then 


$$
\begin{aligned}
\varlimsup_{h \rightarrow 0^{+}}\left(\frac{1}{2 h} \int_{-\infty}^{\infty}\left|\left(\tau_{h}-\tau_{-h}\right) g\right|^{p}\right)^{1 / p} & =\varlimsup_{h \rightarrow 0^{+}}(2 h)^{(p-1) / p}\left\|\frac{1}{2 h}\left(\tau_{h}-\tau_{-h}\right) g\right\|_{p} \\
& =0 \cdot\|A g\|_{p}=0 .
\end{aligned}
$$

Therefore, we have

Proposition 3.4. For $1<p<\infty$, let $f \in L^{p}(\mathbf{R})$ be absolutely continuous and $f^{\prime} \in L^{p}(\mathbf{R})$. Then $\|f\|_{\mathfrak{p} p}=0$.

Let $a<b$; then (3.4) and Proposition 3.4 imply that $\left\|I_{a, b}(f)\right\|_{\gamma_{p}}=0$ for all $f \in L^{p}(\mathbf{R}), 1<p<\infty$. From this, we immediately draw the following conclusion:

LEMMA 3.5. Let $1<p<\infty$, let $f, f_{1}, \ldots, f_{k} \in L^{p}(\mathbf{R})$ and let $a_{1}, \ldots, a_{k}$, $b_{1}, \ldots, b_{k} \in \mathbf{R}$ with $a_{n}<b_{n}$. Then

$$
\left\|f-\sum_{n=1}^{k} I_{a_{n}, b_{n}}\left(f_{n}\right)\right\|_{\uparrow \uparrow}=\|f\|_{\uparrow p} .
$$

TheOREM 3.6. For $1<p<\infty$, the normed linear space $\mathfrak{T}(\mathbf{R})$ is complete.

Proof. For convenience, we let $I_{0, b}=I_{b}$. Let $\left\{f_{n}\right\}$ be a Cauchy sequence in $\Upsilon^{p}(\mathbf{R})$; it suffices to show that $\left\{f_{n}\right\}$ has a convergent subsequence. Without loss of generality, we assume that

$$
\left\|f_{n+1}-f_{n}\right\|_{\Upsilon \uparrow} \leqslant 1 / 2^{n+1}
$$

Also, by Theorem 3.3, we may assume that $f_{n} \in L^{p}(\mathbf{R})$. For $n>1$, select a decreasing sequence of positive numbers $\left\{h_{n}\right\}$ in $(0,1)$ such that $\lim _{n \rightarrow \infty} h_{n}=0$ and for $0<h<h_{n}$,

$$
(1 / 2 h)^{1 / p}\left\|\left(\tau_{h}-\tau_{-h}\right)\left(f_{n+1}-f_{n}\right)\right\|_{p} \leqslant 1 / 2^{n} .
$$

Define $\varepsilon_{1}=1$ and $\varepsilon_{n}, n \geqslant 2$ satisfying

$$
\begin{gathered}
\left\{\varepsilon_{n}\right\} \searrow 0 \text { as } n \rightarrow \infty \\
\left\|\left(I_{\varepsilon_{n}}-1\right) f_{1}\right\|_{p} \leqslant\left(2 h_{n}\right)^{1 / p} / 2^{n} \\
\left\|\left(I_{e_{n}}-1\right)\left(f_{m}-f_{l}\right)\right\|_{p}<\left(2 h_{n}\right)^{1 / p} / 2^{n}, \quad m=n, n-1 \text { and } l=1, \ldots, m-1 .
\end{gathered}
$$

((3.7) and (3.8) follow from (3.2).) For any positive integers $j<k$,

$$
\begin{aligned}
&\left\|\sum_{n=j}^{k}\left(I_{e_{n+1}}-I_{e_{n}}\right) f_{n}\right\|_{p}<\sum_{n=j}^{k}(\left\|\left(I_{e_{n+1}}-1\right)\left(f_{n}-f_{1}\right)\right\|_{p} \\
&+\left\|\left(I_{e_{n}}-1\right)\left(f_{n}-f_{1}\right)\right\|_{p} \\
&\left.+\left\|\left(I_{e_{n+1}}-1\right) f_{1}\right\|_{p}+\left\|\left(I_{e_{n}}-1\right) f_{1}\right\|_{p}\right) \\
&<1 / 2^{j-4} \quad(\text { by }(3.7) \text { and }(3.8)) .
\end{aligned}
$$


Therefore, the sequence

$$
\left\{I_{e_{1}}\left(f_{1}\right)+\sum_{n=1}^{k}\left(I_{e_{n+1}}-I_{e_{n}}\right)\left(f_{n}\right)\right\}_{k=1}^{\infty}
$$

is Cauchy in $L^{p}(\mathbf{R})$ and converges to, say, $f \in L^{p}(\mathbf{R})$, i.e.,

$$
f=I_{e_{1}}\left(f_{1}\right)+\sum_{n=1}^{\infty}\left(I_{e_{n+1}}-I_{e_{n}}\right) f_{n} \text { in } L^{p}(\mathbf{R}) .
$$

Note that for each $g \in L^{p}(\mathbf{R})$, by (3.2) and the telescoping of the terms,

$$
g=I_{e_{1}}(g)+\sum_{n=1}^{\infty}\left(I_{e_{n+1}}-I_{e_{n}}\right) g \text { in } L^{p}(\mathbf{R}) .
$$

We will estimate the term $\left\|f-f_{k}\right\|_{\uparrow p}$ :

$$
\begin{aligned}
\left\|f-f_{k}\right\|_{\uparrow p} & =\varlimsup_{h \rightarrow 0^{+}}\left(\frac{1}{2 h}\right)^{1 / p}\left\|\left(\tau_{h}-\tau_{-h}\right)\left(f-f_{k}\right)\right\|_{p} \\
& =\varlimsup_{h \rightarrow 0^{+}}\left(\frac{1}{2 h}\right)^{1 / p}\left\|\left(\tau_{h}-\tau_{-h}\right)\left(I_{\varepsilon_{1}}\left(f_{1}-f_{k}\right)+\sum_{n=1}^{\infty}\left(I_{\varepsilon_{n+1}}-I_{\varepsilon_{n}}\right)\left(f_{n}-f_{k}\right)\right)\right\|_{p} \quad \text { (by (3.9), (3.10)) } \\
& =\varlimsup_{h \rightarrow 0^{+}}\left(\frac{1}{2 h}\right)^{1 / p}\left\|\left(\tau_{h}-\tau_{-h}\right) \sum_{n=k+1}^{\infty}\left(I_{e_{n+1}}-I_{e_{n}}\right)\left(f_{n}-f_{k}\right)\right\|_{p}
\end{aligned}
$$

(by Lemma 3.5)

For abbreviation, for any positive integers $r<l$ and $h>0$, let

$$
A_{h, r, l}=\left(\frac{1}{2 h}\right)^{1 / p}\left\|\left(\tau_{h}-\tau_{-h}\right)\left(\sum_{n=r+1}^{l}\left(I_{e_{n+1}}-I_{e_{n}}\right)\left(f_{n}-f_{k}\right)\right)\right\|_{p} .
$$

Fix $h \in\left(0, h_{k}\right)$ and let $q>k$ be the unique integer such that $h_{q} \leqslant h<h_{q-1}$. For $l>q$, we have $A_{h, k, l} \leqslant A_{h, k, q}+A_{h, q, l}$. Observe that

$$
\begin{aligned}
A_{h, k, q} & =\left(\frac{1}{2 h}\right)^{1 / p}\left\|\left(\tau_{h}-\tau_{-h}\right) \sum_{n=k+1}^{q}\left(I_{e_{n+1}}-I_{e_{n}}\right) \sum_{j=k+1}^{n}\left(f_{j}-f_{j-1}\right)\right\|_{p} \\
& =\left(\frac{1}{2 h}\right)^{1 / p}\left\|\left(\tau_{h}-\tau_{-h}\right) \sum_{j=k+1}^{q}\left(I_{e_{q+1}}-I_{\xi_{j}}\right)\left(f_{j}-f_{j-1}\right)\right\|_{p}
\end{aligned}
$$

(changing order of summation and adding up telescoping terms)

$$
\begin{aligned}
& <\left(\frac{1}{2 h}\right)^{1 / p} \sum_{j=k+1}^{q}\left\|\left(I_{e_{q+1}}-I_{\xi_{j}}\right)\left(\tau_{h}-\tau_{-h}\right)\left(f_{j}-f_{j-1}\right)\right\|_{p} \\
& <2\left(\frac{1}{2 h}\right)^{1 / p} \sum_{j=k+1}^{q}\left\|\left(\tau_{h}-\tau_{-h}\right)\left(f_{j}-f_{j-1}\right)\right\|_{p} \quad(\text { by }(3.3)) \\
& <1 / 2^{k-2} \quad(\text { by }(3.5))
\end{aligned}
$$


and

$$
\begin{aligned}
A_{h, q, l} & <\sum_{n=q+1}^{l}\left(\frac{1}{2 h}\right)^{1 / p}\left\|\left(\tau_{h}-\tau_{-h}\right)\left(I_{e_{n+1}}-I_{e_{n}}\right)\left(f_{n}-f_{k}\right)\right\|_{p} \\
& <2 \sum_{n=q+1}^{l}\left(\frac{1}{2 h_{n}}\right)^{1 / p}\left\|\left(I_{e_{n+1}}-I_{e_{n}}\right)\left(f_{n}-f_{k}\right)\right\|_{p} \quad \text { (by (3.3)) } \\
& <2 \sum_{n=q+1}^{l}\left(\frac{1}{2 h_{n}}\right)^{1 / p}\left(\left\|\left(I_{e_{n+1}}-1\right)\left(f_{n}-f_{k}\right)\right\|_{p}+\left\|\left(I_{e_{n}}-1\right)\left(f_{n}-f_{k}\right)\right\|_{p}\right) \\
& <1 / 2^{k-2} \text { (by (3.8)). }
\end{aligned}
$$

We have shown that for any integer $k$, for any $h \in\left(0, h_{k}\right)$, and for large integer $l$ (i.e., $l>q$ as defined previously), $A_{h, k, l}<1 / 2^{k-3}$. This implies that

$$
\left\|f-f_{k}\right\|_{\uparrow} \leqslant 1 / 2^{k-3}
$$

and hence $\left\{f_{k}\right\}$ converges to $f$ in $\mathcal{W}^{p}(\mathbf{R})$.

We conclude this section by considering a related space, $\Upsilon^{p}(C), C=[0,2 \pi]$, which consists of those Borel measurable functions on $\mathbf{R}$ with period $2 \pi$ and

$$
\|f\|=\varlimsup_{h \rightarrow 0^{+}}\left(\frac{1}{h} \int_{0}^{2 \pi}\left|\Delta_{h} f\right|^{p}\right)^{1 / p}<\infty .
$$

By identifying functions whose differences have zero norm, it is easy to show that $\tau^{p}(C)$ is a normed linear space. If we consider functions on $C$ as $2 \pi$-periodic functions on $\mathbf{R}$, we can prove the following (compare this to Theorem 3.3):

TheOREM 3.7. For $1<p<\infty, \mathfrak{T}^{p}(C) \subseteq L^{p}(C)$.

REMARK. In [6], Hardy and Littlewood proved that $\Upsilon^{p}(C) \cap L^{1}(C) \subseteq L^{p}(C), 1$ $<p<\infty$.

The proof depends on two results due to Carroll [4] and Boas [2]: Let $\Delta L^{p}(C)$ denote the set of functions $f$ (not necessary measurable) on $C$ such that $\Delta_{h} f=\left(\tau_{h}\right.$ - 1) $f$ is in $L^{p}(C)$. Carroll proved that if $f \in \Delta L^{p}(C), 1<p<\infty$, then $f$ admits a decomposition $f=g+H+S$ where $g \in L^{p}(C)$,

$$
H(h)=\int_{0}^{2 \pi} \Delta_{h} f(x) d x
$$

which is additive on $C$, and $\Delta_{h} S(x)=0$ for almost all $x$. Moreover, Boas proved that if the above $S$ is measurable, then $S$ is constant a.e.

Proof of Theorem 3.7. Let $f \in \mathcal{V}^{p}(C)$. Then $\Delta_{h} f \in L^{p}(C)$. Let $g, H$ and $S$ be defined as above. Since $f$ is measurable and $\Delta_{h} f$ is integrable on $C$, Tonelli's theorem applied to $\left(\Delta_{h} f\right)^{+}$and $\left(\Delta_{h} f\right)^{-}$shows that $H$ is measurable. In addition, the expression for $H$ shows that $H$ is additive and periodic on $\mathbf{R}$, so that it is identically zero. This implies that $S$ is measurable and hence $S$ is a constant a.e. Therefore $f=g+C$ a.e. and $f \in L^{p}(\mathbf{R})$.

By using Theorem 3.7 and the same argument as in Theorem 3.6, we obtain

TheOREM 3.8. For $1<p<\infty, \mathfrak{V}^{p}(C)$ is a Banach space. 
4. Tauberian theorems. In proving the identity (1.1), Wiener introduced a fairly general form of Tauberian theorem which applies to functions in $\mathscr{W}^{2}(\mathbf{R})$. In this section, we will consider two similar types of theorems which apply to functions in $\Re^{p}(\mathbf{R})$.

Let $g$ be a function of bounded variation on $[a, b]$. For all $x \in(a, b]$, let $\mu_{g}(a, x]=g(x+)-g(a+)$. Then it is well known that $\mu_{g}$ has a unique countably additive, regular extension to the $\sigma$-algebra of Borel subsets of $[a, b]$. The following integration by parts holds.

LEMMA 4.1. Let $f, g$ be measurable functions on $[a, b]$ such that $f$ is integrable and $g$ is of bounded variation. Then

$$
\int_{a}^{b} f(x) g(x) d x=\left(\int_{a}^{b} f(x) d x\right) g(b)-\int_{a}^{b}\left(\int_{a}^{x} f(t) d t\right) d \mu_{g}(x) .
$$

Proof. The result follows by applying the Fubini theorem to the right-hand side of the identity

$$
\int_{a}^{b} f(x) g(x) d x=\int_{a}^{b} f(x)\left(\int_{a}^{x} d \mu_{g}(t)+g(a+)\right) d x
$$

Let $S^{+}$denote the set of positive Borel measurable functions on $[0, \infty]$ such that

$$
\sup _{1<T} \frac{1}{T} \int_{0}^{T} f(x) d x \leqslant 1 .
$$

For any $T, \alpha>0$ and for any $f \in S^{+}$, the substitution $x=t / T$ shows that

$$
\int_{0}^{\alpha} f(T x) d x=\alpha\left(\frac{1}{\alpha T} \int_{0}^{\alpha T} f(t) d t\right)<\alpha .
$$

Proposition 4.2. Let $h$ be a positive decreasing integrable function on $[0, \infty)$. Then

(i) $\int_{0}^{\infty} f(T x) h(x) d x \leqslant \int_{0}^{\infty} h(x) d x$ for all $f \in S^{+}, T>1$,

(ii) $\lim _{\alpha \rightarrow \infty} \int_{\alpha}^{\infty} f(T x) h(x) d x=0$ uniformly for all $f \in S^{+}, T>1$.

Proof. (i) Note that because $h$ is a decreasing function, the corresponding measure $\mu_{h}$ is negative. Hence for any $\beta>0, f \in S^{+}$,

$$
\begin{aligned}
\int_{0}^{\beta} f(T x) h(x) d x & =\left(\int_{0}^{\beta} f(T x) d x\right) h(\beta)-\int_{0}^{\beta}\left(\int_{0}^{x} f(T t) d t\right) d \mu_{h}(x) \\
& \leqslant \beta h(\beta)-\int_{0}^{\beta} x d \mu_{h}=\int_{0}^{\beta} h(x) d x .
\end{aligned}
$$

Letting $\beta \rightarrow \infty$, we obtain (i). To prove (ii), we observe that by (i), for each $f \in S^{+}, T>0, \int_{0}^{\infty} f(T x) h(x) d x<\infty$; hence

$$
\lim _{\alpha \rightarrow \infty} \int_{\alpha}^{\infty} f(T x) h(x) d x=0 .
$$

In order to obtain the uniform convergence for $f \in S^{+}$and $T>1$, we let

$$
h_{\alpha}(x)= \begin{cases}h(\alpha), & \text { if } x<\alpha, \\ h(x), & \text { if } x>\alpha .\end{cases}
$$


By applying (i) to $h_{\alpha}$, we have for $f \in S^{+}, T>1$,

$$
\begin{aligned}
\int_{\alpha}^{\infty} f(T x) h(x) d x & \leqslant \int_{0}^{\infty} f(T x) h_{\alpha}(x) d x \\
& \leqslant \int_{0}^{\infty} h_{\alpha}(x) d x=\alpha h(\alpha)+\int_{\alpha}^{\infty} h(x) d x .
\end{aligned}
$$

Note that because $h$ is decreasing,

$$
\frac{\alpha}{2} h(\alpha) \leqslant \int_{\alpha / 2}^{\alpha} h(x) d x \rightarrow 0 \quad \text { as } \alpha \rightarrow \infty .
$$

Also,

$$
\int_{\alpha}^{\infty} h(x) d x \rightarrow 0 \text { as } \alpha \rightarrow \infty
$$

This implies that

$$
\lim _{\alpha \rightarrow \infty} \int_{\alpha}^{\infty} f(T x) h(x) d x=0 \quad \text { uniformly for all } f \in S^{+}, T>1
$$

LEMMA 4.3. Let $h$ be a positive continuous function on $[0, \infty)$ and let $\tilde{h}(x)=$ $\sup _{t>x} h(t)$. Suppose that $\tilde{h} \in L^{1}[0, \infty)$ and suppose there exist disjoint intervals $\left(a_{i}, b_{i}\right), i=1, \ldots, k$, in $[0, \infty)$ such that for each $x \in\left(a_{i}, b_{i}\right), h(x)<h\left(b_{i}\right)$. Let

$$
\eta=\sum_{i=1}^{k}\left(h\left(b_{i}\right)\left(b_{i}-a_{i}\right)-\int_{a_{i}}^{b_{i}} h(x) d x\right) .
$$

Then

$$
\sup _{f \in S^{+}}\left(\varlimsup_{T \rightarrow \infty} \int_{0}^{\infty} f(T x) h(x) d x\right) \geqslant \int_{0}^{\infty} h(x) d x+\eta
$$

Proof. It suffices to show that for any $0<\varepsilon<\eta$, there exists an $f \in S^{+}$such that

$$
\varlimsup_{T \rightarrow \infty} \int_{0}^{\infty} f(T x) h(x) d x \geqslant \int_{0}^{\infty} h(x) d x+(\eta-\varepsilon) .
$$

We will consider the case $k=1$ only. The case $k>1$ follows from the same idea of proof. We write $a_{1}=a$ and $b_{1}=b$ and without loss of generality assume that $a>0$. Otherwise, let $\left\{\tilde{a}_{n}\right\} \searrow 0$ and let

$$
\eta_{n}=h(b)\left(b-\tilde{a}_{n}\right)-\int_{\tilde{a}_{n}}^{b} h(x) d x
$$

then $\left\{\eta_{n}\right\} \rightarrow \eta$. We can prove $(4.1)$ for $\left(\tilde{a}_{n}, b\right)$ and $\eta_{n}$.

Since $h$ is continuous, for $\varepsilon>0$, we can find $0<\varepsilon_{1}, 0<\delta<\varepsilon / 8$ such that $|x-b|<\delta$ implies that $|h(x)-h(b)|<\varepsilon_{1}$ and

$$
\eta-\frac{\varepsilon}{2}<\left(h(b)-\varepsilon_{1}\right)(b+\delta-a)-\int_{a}^{b+\delta} h(x) d x .
$$


Since $\tilde{h}$ is decreasing and integrable, Proposition 4.2(ii) implies that there exists an $\alpha>b+\delta$ such that

$$
\int_{\alpha}^{\infty} p(T x) h(x) d x \leqslant \int_{\alpha}^{\infty} p(T x) \tilde{h}(x) d x<\delta \quad \forall p \in S^{+}, T \geqslant 1 .
$$

In particular,

$$
\int_{\alpha}^{\infty} h(x) d x<\delta
$$

Let $\left\{\delta_{n}\right\}$ be a sequence of positive numbers such that $\sum_{n=1}^{\infty} \delta_{n}<\delta$ and let $M$ be the upper bound of $h$. Let $T_{1} \geqslant 1$ and let

$$
f_{1}(x)= \begin{cases}1, & 0 \leqslant x<\alpha T_{1}, \\ 0, & \alpha T_{1} \leqslant x .\end{cases}
$$

Suppose we have chosen $T_{n-1}, f_{n-1}$. Select $T_{n}$ such that

$$
T_{n}>\max \left\{\frac{\alpha}{a} T_{n-1}, \frac{\alpha M}{\delta_{n}} T_{n-1}\right\}
$$

and define

$$
f_{n}(x)= \begin{cases}0, & 0 \leqslant x<\alpha T_{n-1}, \\ 1, & \alpha T_{n-1} \leqslant x<a T_{n}, \\ 0, & a T_{n} \leqslant x<b T_{n}, \\ \frac{b+\delta-a}{\delta}, & b T_{n} \leqslant x<(b+\delta) T_{n}, \\ 1, & (b+\delta) T_{n} \leqslant x<\alpha T_{n}, \\ 0, & \alpha T_{n} \leqslant x .\end{cases}
$$

Note that the functions $\left\{f_{n}\right\}$ have disjoint supports. Let $f=\sum_{n=1}^{\infty} f_{n}$. It is easy to show that

$$
\frac{1}{T} \int_{0}^{T} f(x) d x=1 \text { for } T \in[1, \infty) \backslash \bigcup_{n=2}^{\infty}\left(a T_{n},(b+\delta) T_{n}\right)
$$

and

$$
\frac{1}{T} \int_{0}^{T} f(x) d x<1 \quad \text { for } T \in \bigcup_{n=2}^{\infty}\left(a T_{n},(b+\delta) T_{n}\right) .
$$

Hence $f \in S^{+}$. We will show that

$$
\varlimsup_{n \rightarrow \infty} \int_{0}^{\infty} f\left(T_{n} x\right) h(x) d x>\int_{0}^{\infty} h(x) d x+(\eta-\varepsilon) .
$$


For $n>1$,

$$
\begin{aligned}
\mid \int_{0}^{\infty} f\left(T_{n} x\right) & h(x) d x-\int_{0}^{\alpha} f_{n}\left(T_{n} x\right) h(x) d x \mid \\
\leqslant & \left|\int_{0}^{\alpha} f\left(T_{n} x\right) h(x) d x-\int_{0}^{\alpha} f_{n}\left(T_{n} x\right) h(x) d x\right|+\delta \\
\leqslant & \sum_{i=1}^{n-1} \int_{0}^{\alpha} f_{i}\left(T_{n} x\right) h(x) d x+\sum_{i=n+1}^{\infty} \int_{0}^{\alpha} f_{i}\left(T_{n} x\right) h(x) d x+\delta \\
\leqslant & \sum_{i=1}^{n-1} \frac{M}{T_{n}} \int_{0}^{\alpha T_{i}} f_{i}(x) d x+0+\delta \\
& <\sum_{i=1}^{n-1} \frac{M}{T_{n}} \alpha T_{i}+\delta<\sum_{i=1}^{n-1} \delta_{i+1}+\delta<2 \delta
\end{aligned}
$$

and

$$
\begin{aligned}
\int_{0}^{\alpha} f_{n} & \left(T_{n} x\right) h(x) d x \\
& =\int_{\alpha T_{n-1} / T_{n}}^{a} h(x) d x+\int_{b}^{b+\delta} \frac{(b+\delta)-a}{\delta} h(x) d x+\int_{b+\delta}^{\alpha} h(x) d x \\
& >\int_{0}^{a} h(x) d x-M \frac{\delta_{n}}{M}+(b+\delta-a)\left(h(b)-\varepsilon_{1}\right)+\int_{b+\delta}^{\alpha} h(x) d x \\
& >\int_{0}^{\alpha} h(x) d x-\delta_{n}+\left(\eta-\frac{\varepsilon}{2}\right) \quad(\text { by }(4.2)) \\
& >\int_{0}^{\infty} h(x) d x+\eta-\left(2 \delta+\frac{\varepsilon}{2}\right) .
\end{aligned}
$$

Combining the above two estimations we have, for $n>1$,

$$
\int_{0}^{\infty} f\left(T_{n} x\right) h(x) d x>\int_{0}^{\infty} h(x) d x+(\eta-\varepsilon) .
$$

Proposition 4.4. Let $h$ be a positive continuous function on $[0, \infty)$. Let $\tilde{h}(x)=$ $\sup _{t>x} h(x)$ and assume that $\tilde{h}$ is integrable. Then

$$
\sup _{f \in S^{+}}\left(\varlimsup_{T \rightarrow \infty} \int_{0}^{\infty} f(T x) h(x) d x\right)=\int_{0}^{\infty} \tilde{h}(x) d x .
$$

Proof. Since $\tilde{h}$ is decreasing, by Proposition 4.2(i),

$$
\begin{aligned}
\sup _{f \in S^{+}}\left(\varlimsup_{T \rightarrow \infty} \int_{0}^{\infty} f(T x) h(x) d x\right) & <\sup _{f \in S^{+}}\left(\varlimsup_{T \rightarrow \infty} \int_{0}^{\infty} f(T x) \tilde{h}(x) d x\right) \\
& <\int_{0}^{\infty} \tilde{h}(x) d x .
\end{aligned}
$$

We will prove the reverse inequality. That $\tilde{h}$ is integrable yields an $\alpha>0$ such that $\int_{\alpha}^{\infty} \tilde{h}(x) d x<\varepsilon$. Since $h$ is continuous, so is $\tilde{h}$; hence the set $\{x \in(0, \alpha): \tilde{h}(x)>$ $h(x)\}$ is the union of a (finite or infinite) sequence of disjoint open intervals 
$\left\{\left(a_{i}, b_{i}\right)\right\}$ in $(0, \alpha)$. Let $1 \leqslant r \leqslant \infty$ be the number of such intervals. Note that $\tilde{h}$ is constant on each $\left(a_{i}, b_{i}\right)$. It follows that

$$
\int_{0}^{\alpha} \tilde{h}(x) d x=\int_{0}^{\alpha} h(x) d x+\sum_{i=1}^{r}\left(h\left(b_{i}\right)\left(b_{i}-a_{i}\right)-\int_{a_{i}}^{b_{i}} h(x) d x\right) .
$$

Lemma 4.3 implies that for any integer $k, 0 \leqslant k \leqslant r$,

$$
\begin{aligned}
\sup _{f \in S^{+}}\left(\varlimsup_{T \rightarrow \infty} \int_{0}^{\infty}\right. & f(T x) h(x) d x) \\
& >\int_{0}^{\alpha} h(x) d x+\sum_{i=1}^{k}\left(h\left(b_{i}\right)\left(b_{i}-a_{i}\right)-\int_{a_{i}}^{b_{i}} h(x) d x\right) .
\end{aligned}
$$

Hence,

$$
\sup _{f \in S^{+}}\left(\varlimsup_{T \rightarrow \infty} \int_{0}^{\infty} f(T x) h(x) d x\right)>\int_{0}^{\alpha} \tilde{h}(x) d x
$$

and the proof is completed by observing that $\int_{\alpha}^{\infty} \tilde{h}(x) d x<\varepsilon$.

Let $\mathfrak{K}^{+}$denote the class of positive Borel measurable functions $f$ on $[0, \infty)$ such that $\varlimsup_{T \rightarrow \infty}(1 / T) \int_{0}^{T} f(x) d x<\infty$. The following is the first main result of this section.

TheORem 4.5. Let $h$ be a positive, continuous function on $[0, \infty)$. Assume that (i) $\tilde{h}(x)=\sup _{t>x} h(t)$ is integrable and $C_{1}=\int_{0}^{\infty} \tilde{h}(x) d x$, and (ii) $f \in \mathscr{T}^{+}$. Then

$$
\varlimsup_{T \rightarrow \infty} \int_{0}^{\infty} f(T x) h(x) d x \leqslant C_{1} \varlimsup_{T \rightarrow \infty} \frac{1}{T} \int_{0}^{T} f(x) d x .
$$

Moreover, $C_{1}$ is the best estimation of the inequality for the class of functions $f$ in $\mathfrak{R}^{+}$.

Proof. For any function $p$ which is integrable on $[0, \rho)$ and vanishes on $[\rho, \infty)$,

$$
\begin{aligned}
\lim _{T \rightarrow \infty} \int_{0}^{\infty} p(T x) h(x) d x & =\lim _{T \rightarrow \infty} \frac{1}{T} \int_{0}^{\rho} p(t) h\left(\frac{t}{T}\right) d t \\
& \leqslant\left(\lim _{T \rightarrow \infty} \frac{1}{T}\right)\left(M \int_{0}^{\rho} p(t) d t\right)=0
\end{aligned}
$$

where $M=\sup _{t>0} h(t)$. Let $f_{\rho}=f \chi_{[\rho, \infty)}, \rho>1$. It follows from (4.3) that

$$
\varlimsup_{T \rightarrow \infty} \int_{0}^{\infty} f(T x) h(x) d x=\varlimsup_{T \rightarrow \infty} \int_{0}^{\infty} f_{\rho}(T x) h(x) d x .
$$

Applying Proposition 4.4 with $\left(\sup _{T>\rho}(1 / T) \int_{0}^{T} f\right)^{-1} f_{\rho} \in S^{+}$, we get

$$
\varlimsup_{T \rightarrow \infty} \int_{0}^{\infty} f_{\rho}(T x) h(x) d x \leqslant C_{1} \sup _{T>\rho} \frac{1}{T} \int_{0}^{T} f(x) d x, \quad \rho>0 .
$$

Hence by (4.4),

$$
\varlimsup_{T \rightarrow \infty} \int_{0}^{\infty} f(T x) h(x) d x \leqslant C_{1} \varlimsup_{T \rightarrow \infty} \frac{1}{T} \int_{0}^{T} f(x) d x .
$$


To prove the last assertion, it suffices to show that for any $\varepsilon>0$, there exists an $f_{\varepsilon}$ such that

$$
\varlimsup_{T \rightarrow \infty} \frac{1}{T} \int_{0}^{T} f_{\varepsilon}(x) d x \leqslant 1 \text { and } \varlimsup_{T \rightarrow \infty} \int_{0}^{\infty} f_{\varepsilon}(T x) h(x) d x \leqslant\left(C_{1}-\varepsilon\right) .
$$

But this inequality is obvious from Proposition 4.4.

Our second main result in this section is:

THEOREM 4.6. Let $h$ be a positive continuous function on $[0, \infty)$ such that $\tilde{h}(x)=$ $\sup _{t>x} h(t)$ is integrable. Suppose (i) there is an $x_{0}$ which satisfies $x_{0} h\left(x_{0}\right)=$ $\max _{x>0} x h(x)=C_{2}$ and $h(x) \geqslant h\left(x_{0}\right)$ for all $x$ in $\left[0, x_{0}\right]$, (ii) $f \in \Re^{+}$. Then

$$
C_{2} \varlimsup_{T \rightarrow \infty} \frac{1}{T} \int_{0}^{T} f(x) d x \leqslant \varlimsup_{T \rightarrow \infty} \int_{0}^{\infty} f(T x) h(x) d x .
$$

Moreover, $C_{2}$ is the best estimation of the inequality for $f \in \mathfrak{K}^{+}$.

Proof. Let $f$ be given as above; then

$$
\begin{aligned}
C_{2} \frac{1}{T x_{0}} \int_{0}^{T x_{0}} f(x) d x=\frac{1}{T} \int_{0}^{T x_{0}} f(x) h\left(x_{0}\right) d x \\
\quad \leqslant \frac{1}{T} \int_{0}^{T x_{0}} f(x) h\left(\frac{x}{T}\right) d x \quad\left(\text { since } h(y)>h\left(x_{0}\right) \forall y \in\left[0, x_{0}\right]\right) \\
\quad=\int_{0}^{x_{0}} f(T x) h(x) d x \\
\quad \leqslant \int_{0}^{\infty} f(T x) h(x) d x .
\end{aligned}
$$

By taking limit supremum on both sides, the first part of the theorem follows.

To prove the second part, we will construct, for a given $0<\varepsilon<1$, a positive $f$ such that

$$
\varlimsup_{T \rightarrow \infty} \frac{1}{T} \int_{0}^{T} f(x) d x=1 \text { and } \varlimsup_{T \rightarrow \infty} \int_{0}^{\infty} f(T x) h(x) d x<C_{2}+\varepsilon .
$$

We will need the following statement, where the proof depends on the uniform continuity of $h$ : for any $a>0$,

$$
\begin{aligned}
\lim _{\delta \rightarrow 0^{+}} \frac{x_{0}+\delta}{\delta} \int_{\beta x_{0}}^{\beta\left(x_{0}+\delta\right)} h(x) d x=\left(\beta x_{0}\right) h\left(\beta x_{0}\right) \\
\text { uniformly for } 0 \leqslant \beta \leqslant a .
\end{aligned}
$$

For any $0<\varepsilon<1$, we choose $\alpha>x_{0}$ such that

$$
\int_{\alpha}^{\infty} p(T x) \tilde{h}(x) d x<\frac{\varepsilon}{8} \quad \forall p \in S^{+} \text {and } T \geqslant 1
$$

(Proposition 4.2(ii)). Since $x_{0} h\left(x_{0}\right)=\max _{x>0} x h(x)$, by (4.5), there exists $0<\delta<$ $\alpha-x_{0}$ which satisfies

$$
\frac{x_{0}+\delta}{\delta} \int_{\beta x_{0}}^{\beta\left(x_{0}+\delta\right)} h(x) d x<x_{0} h\left(x_{0}\right)+\frac{\varepsilon}{4} \quad \forall 0<\beta<\frac{\alpha}{x_{0}} .
$$


Let $T_{1}>1$ and select $T_{n}>T_{n-1}$ so that

$$
\frac{T_{n-1}}{T_{n}}<\frac{x_{0}}{\alpha}, \quad \frac{1}{T_{n}} \sum_{i=1}^{n-1} T_{i}<\frac{1}{n}
$$

and

$$
\sum_{i=1}^{n-1} \int_{0}^{x_{0} T_{i} / T_{n}} h(x) d x<\frac{\delta}{x_{0}+\delta} \frac{\varepsilon}{4}
$$

Let

$$
f_{n}=\frac{x_{0}+\delta}{\delta} \chi_{\left[x_{0} T_{n},\left(x_{0}+\delta\right) T_{n}\right]} \text { and } f=\sum_{n=1}^{\infty} f_{n} .
$$

Note that the functions $\left\{f_{n}\right\}$ have disjoint supports. For any $n$,

$$
\begin{aligned}
\frac{1}{\left(x_{0}+\delta\right) T_{n}} & \int_{0}^{\left(x_{0}+\delta\right) T_{n}} f(x) d x \\
& =\frac{1}{\left(x_{0}+\delta\right) T_{n}}\left(\left(x_{0}+\delta\right) T_{n}+\sum_{i=1}^{n-1}\left(x_{0}+\delta\right) T_{i}\right) \\
& =1+\frac{1}{T_{n}} \sum_{i=1}^{n-1} T_{i} \leqslant 1+\frac{1}{n} .
\end{aligned}
$$

Since $(1 / T) \int_{0}^{T} f(x) d x$ has a local maximum at each $\left(x_{0}+\delta\right) T_{n}$, we have

$$
\sup _{1<T} \frac{1}{T} \int_{0}^{T} f(x) d x \leqslant 2 \text { and } \varlimsup_{T \rightarrow \infty} \frac{1}{T} \int_{0}^{T} f(x) d x=1 .
$$

Now for any $T \geqslant 1$, there exists an $n$ such that $x_{0} T_{n} \leqslant \alpha T<x_{0} T_{n+1}$. Hence $0<T_{n} / T<\alpha / x_{0}$ and

$$
\begin{aligned}
\int_{0}^{\infty} f(T x) h(x) d x & \leqslant \int_{0}^{\alpha} f(T x) h(x) d x+\frac{\varepsilon}{4} \quad \text { (by (4.6) and (4.8)) } \\
& =\sum_{i=1}^{n} \int_{0}^{\alpha} f_{i}(T x) h(x) d x+\frac{\varepsilon}{4} \\
& \leqslant \sum_{i=1}^{n} \int_{x_{0} T_{i} / T}^{\left(x_{0}+\delta\right) T_{i} / T} \frac{x_{0}+\delta}{\delta} h(x) d x+\frac{\varepsilon}{4} \\
& <\frac{x_{0}+\delta}{\delta} \int_{x_{0} T_{n} / T}^{\left(x_{0}+\delta\right) T_{n} / T} h(x) d x+\frac{\varepsilon}{2} \quad(\text { by (4.7)) } \\
& \leqslant x_{0} h\left(x_{0}\right)+\varepsilon / 4+\varepsilon / 2 \quad(\text { by (4.6)) } \\
& <C_{2}+\varepsilon .
\end{aligned}
$$

The proof is complete by taking the limit supremum on $T$.

We remark that the function $h(x)=\left|2 \sin ^{p} x / \pi x^{p}\right|, x>0,1<p<\infty$, satisfies the hypotheses in Theorems 4.5 and 4.6. We leave the simple verification to the reader. This function will be considered throughout the rest of the paper. 


\section{The Wiener transformation $W$.}

A. The isomorphic nature of $W$ on $\mathfrak{N}^{2}(\mathbf{R})$.

Proposition 2.1 implies that $M^{2}(\mathbf{R}) \subseteq L^{2}\left(\mathbf{R}, d x /\left(1+x^{2}\right)\right)$; hence for $f \in M^{2}(\mathbf{R})$, the integral

$$
\int_{-\infty}^{-1}+\int_{1}^{\infty} \frac{|f(x)|^{2}}{x^{2}} d x
$$

exists. This implies that

$$
\int_{-\infty}^{-1}+\int_{1}^{\infty} \frac{f(x) e^{-i u x}}{-i x} d x
$$

converges in mean square. In [18], Wiener defined the following transformation $W$ : for $f \in M^{2}(\mathbf{R})$, let $W(f)=g$ where

$$
g(u)=\frac{1}{2 \pi}\left(\int_{-\infty}^{-1}+\int_{1}^{\infty} f(x) \frac{e^{-i u x}}{-i x} d x+\int_{-1}^{1} f(x) \frac{e^{-i u x}-1}{-i x} d x\right)
$$

We call $W$ the Wiener transformation. Now, for $h>0$,

$$
\begin{aligned}
\left(\tau_{h} g-\tau_{-h} g\right)(u) & =\frac{1}{2 \pi} \int_{-\infty}^{\infty} f(x) \frac{e^{i h x}-e^{-i h x}}{i x} e^{-i u x} d x \\
& =\frac{1}{2 \pi} \int_{-\infty}^{\infty} f(x) \frac{2 \sin (h x)}{x} e^{-i u x} d x
\end{aligned}
$$

Thus $\tau_{h} g-\tau_{-h} g$ is the Fourier transformation of

$$
\sqrt{\frac{2}{\pi}} f(x) \frac{\sin (h x)}{x}
$$

and the Plancherel theorem implies

$$
\frac{1}{2 h} \int_{-\infty}^{\infty}|g(u+h)-g(u-h)|^{2} d u=\frac{1}{h} \int_{-\infty}^{\infty}|f(x)|^{2} \frac{\sin ^{2} h x}{\pi x^{2}} d x
$$

Hence

$$
\begin{aligned}
\|W(f)\|_{\mathbb{F}^{2}}^{2} & =\varlimsup_{h \rightarrow 0^{+}} \frac{1}{h} \int_{-\infty}^{\infty}|f(x)|^{2} \frac{\sin ^{2} h x}{\pi x^{2}} d x \\
& =\varlimsup_{T \rightarrow \infty} \int_{-\infty}^{\infty}|f(T x)|^{2} \frac{\sin ^{2} x}{\pi x^{2}} d x .
\end{aligned}
$$

Letting $h(x)=\left(2 \sin ^{2} x\right) / \pi x^{2}, x>0$, and $f^{\prime}(x)=\frac{1}{2}\left(|f(x)|^{2}+|f(-x)|^{2}\right), x>0, f \in$ $M^{2}(\mathbf{R})$, Theorem 4.5 and (5.1) imply that $W(f) \in \mathcal{V}^{2}(\mathbf{R})$ and $W(f)=0$ for all $f \in I^{2}(\mathbf{R})$. Since $\pi^{2}(\mathbf{R})=M^{2}(\mathbf{R}) / I^{2}(\mathbf{R})$ (Proposition 2.2), $W$ induces a map from $\mathscr{T}^{2}(\mathbf{R})$ into $\mathscr{V}^{2}(\mathbf{R})$.

THEOREM 5.1 (WIENER [18]). Let $f \in \mathcal{W}^{2}(\mathbf{R}) \subseteq \mathfrak{N}^{2}(\mathbf{R})$. Then $\|W(f)\|_{\mathfrak{q}^{2}}=\|f\|_{\mathfrak{R}^{2}}$.

Our main result is: 
THEOREM 5.2. The Wiener transformation $W$ defines an isomorphism from $\mathfrak{T}^{2}(\mathbf{R})$ onto $\mathcal{T}^{2}(\mathbf{R})$ with

$$
\|W\|=\left(\int_{0}^{\infty} \tilde{h}(x) d x\right)^{1 / 2} \text { and }\left\|W^{-1}\right\|=\left(\max _{x>0} x h(x)\right)^{-1 / 2}
$$

where $h(x)=\left(2 \sin ^{2} x\right) / \pi x^{2}, x>0$, and $\tilde{h}(x)=\sup _{t>x} h(t)$.

Proof. It is easy to show that $h(x)=\left(2 \sin ^{2} x\right) / \pi x^{2}, x>0$, satisfies the hypotheses in Theorems 4.5 and 4.6. By letting

$$
f^{\prime}(x)=\frac{1}{2}\left(|f(x)|^{2}+|f(-x)|^{2}\right), \quad x>0, f \in \mathscr{N}^{2}(\mathbf{R})
$$

the same theorems yield

$$
\begin{aligned}
C_{2} \varlimsup_{T \rightarrow \infty} \frac{1}{2 T} \int_{-T}^{T}|f(x)|^{2} d x & <\varlimsup_{T \rightarrow \infty} \int_{-\infty}^{\infty}|f(T x)|^{2} h(x) d x \\
& <C_{1} \varlimsup_{T \rightarrow \infty} \frac{1}{2 T} \int_{-T}^{T}|f(x)|^{2} d x, \quad f \in \mathfrak{N}^{2}(\mathbf{R}),
\end{aligned}
$$

where $C_{1}=\int_{0}^{\infty} \tilde{h}(x) d x$ and $C_{2}=\max _{x>0} x h(x)$. By (5.1), we have

$$
C_{2}\|f\|_{\mathscr{T}^{2}}^{2} \leqslant\|W(f)\|_{\mathfrak{\vartheta}^{2}}^{2}<C_{1}\|f\|_{\mathscr{T}^{2}}^{2}, \quad f \in \mathscr{T}^{2}(\mathbf{R}) .
$$

Moreover, Theorems 4.5 and 4.6 imply that $C_{1}$ and $C_{2}$ are the best constants to estimate the above inequalities. Hence we conclude that $W$ is an isomorphism from $\mathscr{N}^{2}(\mathbf{R})$ into $\Upsilon^{2}(\dot{\mathbf{R}})$ with

$$
\|W\|=C_{1}^{1 / 2} \text { and }\left\|W^{-1}\right\|=C_{2}^{-1 / 2} .
$$

It remains to show that $W$ is a surjection. Let $g \in \mathcal{T}^{2}(\mathbf{R})$; by Theorem 3.3, we may assume that $g \in L^{2}(\mathbf{R})$. Let $\hat{g}$ be the (inverse) Fourier transformation of $g$, i.e.

$$
\hat{g}(x)=\frac{1}{\sqrt{2 \pi}} \int_{-\infty}^{\infty} g(u) e^{i u x} d u
$$

and let $f(x)=-i \sqrt{2 \pi} x \hat{g}(x), x \in \mathbf{R}$. We claim that (i) $f \in \mathscr{T}^{2}(\mathbf{R})$ and (ii) $W(f)=$ $g$ in $\mathcal{V}^{2}(\mathbf{R})$. To prove (i), note that

$$
\left(\tau_{h}(g)-\tau_{-h}(g)\right)^{\wedge}(x)=\left(e^{-i h x}-e^{i h x}\right) \hat{g}(x)=-2 i(\sin (h x)) \hat{g}(x) .
$$

As $C_{2}=\max _{x>0}\left(2 \sin ^{2} x\right) / \pi x$, Theorem 4.6 applied to $f^{\prime}(x)=\frac{1}{2}\left(|f(x)|^{2}+\right.$ $\left.|f(-x)|^{2}\right), x>0$, yields

$$
\begin{aligned}
C_{2} \varlimsup_{T \rightarrow \infty} \frac{1}{2 T} \int_{-T}^{T}|f(x)|^{2} d x & <\varlimsup_{T \rightarrow \infty} \int_{-\infty}^{\infty}|f(T x)|^{2} \frac{\sin ^{2} x}{\pi x^{2}} d x \\
& =\varlimsup_{h \rightarrow 0^{+}} \frac{1}{h} \int_{-\infty}^{\infty}|-i \sqrt{2 \pi} x \hat{g}(x)|^{2} \frac{\sin ^{2} h x}{\pi x^{2}} d x \\
& =\varlimsup_{h \rightarrow 0^{+}} \frac{1}{h} \int_{-\infty}^{\infty} 2|\hat{g}(x)|^{2} \sin ^{2} h x d x \\
& =\|g\|_{\mathfrak{F}^{2}}^{2} \quad(\text { by }(5.2) \text { and the Plancherel theorem) } \\
& <\infty .
\end{aligned}
$$


Thus $f \in \Re^{2}(\mathbf{R})$. To prove (ii), we observe that

$$
\begin{aligned}
W(f)(u) & =\frac{1}{2 \pi}\left(\int_{-\infty}^{-1}+\int_{1}^{\infty} f(x) \frac{e^{-i u x}}{-i x} d x+\int_{-1}^{1} f(x) \frac{e^{-i u x}-1}{-i x} d x\right) \\
& =\frac{1}{\sqrt{2 \pi}}\left(\int_{-\infty}^{\infty} \hat{g}(x) e^{-i u x} d x-\int_{-1}^{1} \hat{g}(x) d x\right) \\
& =g(u)+C \text { a.e. }
\end{aligned}
$$

where $C=-(1 / 2 \pi) \int_{-1}^{1} \hat{g}(x) d x$. Since constant functions in $\mathcal{T}^{2}(\mathbf{R})$ are equivalent to zero, we have $W(f)=g$ in $\mathcal{V}^{2}(\mathbf{R})$.

B. The nonisometric nature of $W$ on $\left\langle\omega^{2}(\mathbf{R})\right\rangle$.

Let $h(x)=\left(2 \sin ^{2} x\right) / \pi x^{2}, x>0$. It follows from Theorem 5.2 and elementary. calculus that

and

$$
\|W\|=\left(\int_{0}^{\infty} \tilde{h}(x) d x\right)^{1 / 2}>\left(\frac{2}{\pi} \int_{0}^{\infty} \frac{\sin ^{2} x}{x^{2}} d x\right)^{1 / 2}=1
$$

$$
\left\|W^{-1}\right\|=\left(\max _{x>0} \frac{2 \sin ^{2} x}{\pi x}\right)^{-1 / 2}>1 .
$$

In view of the fact that $W$ is an isometry on $\mathscr{W}^{2}(\mathbf{R})$ (Theorem 5.1), Masani [16] asked whether $W$ is an isometry when it is restricted on $\left\langle\mho^{2}(\mathbf{R})\right\rangle$, the closed linear subspace generated by $\mathscr{W}^{2}(\mathbf{R})$.

Let $\mathcal{Q}^{2}(\mathbf{R})$ be the subset of ${ }^{2}(\mathbf{R})$ such that for $g \in \mathcal{Q}^{2}(\mathbf{R})$,

$$
\lim _{h \rightarrow 0^{+}} \frac{1}{2 h} \int_{-\infty}^{\infty}|g(u+h)-g(u-h)|^{2} d u
$$

exists. Theorems 5.1 and 5.2 imply that $W$ is an isomorphism from $\left\langle\mho^{2}(\mathbf{R})\right\rangle$ onto $\left\langle\mathcal{Q}^{2}(\mathbf{R})\right\rangle$. In the following, we will give two examples $l_{1}$ and $l_{2} \in\left\langle\mho^{2}(\mathbf{R})\right\rangle$ with $\left\|l_{1}\right\|=\left\|l_{2}\right\|=1$ and $\left\|W\left(l_{1}\right)\right\|>1$ and $\left\|W\left(l_{2}\right)\right\|<1$. Hence neither $W$ nor $W^{-1}$ can be a contraction on $\left\langle\mho^{2}(\mathbf{R})\right\rangle,\left\langle\vartheta^{2}(\mathbf{R})\right\rangle$ respectively. These answer Masani's question negatively. Both examples refine the functions constructed in the proof of Lemma 4.3 and Theorem 4.6.

EXAMPLE 5.3. There exists an $l_{1}$ in $\left\langle\mho^{2}(\mathbf{R})\right\rangle$ with $\left\|l_{1}\right\|_{\mathscr{T}^{2}}=1$ and $\left\|W\left(l_{1}\right)\right\|_{\mathcal{F}^{2}}>1$.

We will use the same notation as in Lemma 4.3 with $h(x)=\left(2 \sin ^{2} x\right) / \pi x^{2}, k=1$, and $\left[a_{1}, b_{1}\right]=[a, b]$ such that $h(b)=h(a)>h(x), x \in(a, b)$. We assume further that the $\delta$ we choose in Lemma 4.3 satisfies $(b+\delta-a) / \delta=(m+1)^{2}$ for some positive integer $m$. Let $T_{n}, f_{n}$ and $f$ be as in Lemma 4.3. Define for $n>1,1<i \leqslant$ $2 m+1$,

$$
f_{n}^{i}(x)= \begin{cases}0, & 0 \leqslant x<\alpha T_{n-1}, \\ \frac{1}{2}(-1)^{i+1}, & \alpha T_{n-1}<x<a T_{n}, \\ \frac{1}{2}(-1)^{i}, & a T_{n}<x<b T_{n}, \\ \frac{1}{2}, & b T_{n} \leqslant x<(b+\delta) T_{n}, \\ \frac{1}{2}(-1)^{i+1}, & (b+\delta) T_{n}<x<\alpha T_{n}, \\ 0, & \alpha T_{n}<x\end{cases}
$$


Note that $\left(\sum_{i=1}^{2 m+1} f_{n}^{i}(x)+\frac{1}{2}\right)^{2}=f_{n}(x)$. For each $i$, the functions $\left\{f_{n}^{i}\right\}_{i=1}^{\infty}$ have disjoint supports. Let $f^{i}=\sum_{n=1}^{\infty} f_{n}^{i}$. Then $\left|f^{i}\right| \equiv \frac{1}{2}$ and $f^{i}$ is in $\mho^{2}(\mathbf{R})$ for each $i$. Let

$$
l_{1}=\sqrt{2}\left(\sum_{i=1}^{2 m+1} f^{i}+\frac{1}{2}\right) \text {. }
$$

Then $l_{1} \in\left\langle\mho^{2}(\mathbf{R})\right\rangle$ and

$$
\begin{aligned}
\left|l_{1}(x)\right|^{2} & =2\left|\sum_{i=1}^{2 m+1}\left(\sum_{n=1}^{\infty} f_{n}^{i}(x)\right)+\frac{1}{2}\right|^{2} \\
& =2\left|\sum_{i=1}^{2 m+1} f_{k}^{i}(x)+\frac{1}{2}\right|^{2} \quad \text { where } x \in \operatorname{supp}\left(f_{k}\right) \\
& =2 f_{k}(x)=2 f(x)
\end{aligned}
$$

Hence

$$
\left\|l_{1}\right\|_{\mathscr{T} \mathbb{R}^{2}}^{2}=\varlimsup_{T \rightarrow \infty} \frac{1}{2 T} \int_{-T}^{T}\left|l_{1}(x)\right|^{2} d x=\varlimsup_{T \rightarrow \infty} \frac{1}{T} \int_{0}^{T} f(x) d x=1
$$

and by (5.1) and (4.1),

$$
\begin{aligned}
\left\|W\left(l_{1}\right)\right\|_{\mathscr{V}^{2}}^{2} & =\varlimsup_{T \rightarrow \infty} \int_{-\infty}^{\infty}\left|l_{1}(T x)\right|^{2} \frac{\sin ^{2} x}{\pi x^{2}} d x \\
& =\varlimsup_{T \rightarrow \infty} \int_{0}^{\infty} 2|f(T x)|^{2} \frac{\sin ^{2} x}{\pi x^{2}} d x \\
& >\int_{0}^{\infty} \frac{2 \sin ^{2} x}{\pi x^{2}} d x=1 .
\end{aligned}
$$

EXAMPLE 5.4. There exists an $l_{2} \in\left\langle\mathcal{W}^{2}(\mathbf{R})\right\rangle$ such that $\left\|l_{2}\right\|_{\mathscr{R}^{2}}=1$ and $\left\|W\left(l_{2}\right)\right\|_{\mathfrak{F}^{2}}$ $<1$.

We use the same notation as in the proof of Theorem 4.6 with $h(x)=$ $\left(2 \sin ^{2} x\right) / \pi x^{2}$ and $C_{2}=\max _{x>0} x h(x)<1$. We assume that $0<\varepsilon<1-C_{2}$ and let $\delta$ be as in Theorem 4.6 and satisfy the condition:

$$
\frac{x_{0}+\delta}{\delta}=(2 m)^{2} \text { for some integer } m \text {. }
$$

Let $\left\{T_{n}\right\}$ be as in Theorem 4.6 and for $n>1,1 \leqslant i \leqslant 2 m$, define

$$
f_{n}^{i}(x)= \begin{cases}0, & x<x_{0} T_{n-1}, \\ (-1)^{i}, & x_{0} T_{n-1} \leqslant x<x_{0} T_{n} \\ 1, & x_{0} T_{n} \leqslant x<\left(x_{0}+\delta\right) T_{n} \\ 0, & \left(x_{0}+\delta\right) T_{n} \leqslant x .\end{cases}
$$

Then

$$
\left(\sum_{i=1}^{2 m} f_{n}^{i}\right)^{2}=\frac{x_{0}+\delta}{\delta} \chi_{\left[x_{0} T_{n},\left(x_{0}+\delta\right) T_{n}\right]}
$$


Note that for each fixed $i$, the supports of the functions $\left\{f_{n}^{i}\right\}_{n=1}^{\infty}$ are disjoint. Let $f^{i}=\sum_{n=1}^{\infty} f_{n}^{i}$; then $\left|f^{i}\right| \equiv 1$ and $f^{i} \in W^{2}(\mathbf{R})$. Let $l_{2}=\sqrt{2} \sum_{i=1}^{2 m} f^{i}$. It follows that $l_{2} \in\left\langle W^{2}(\mathbf{R})\right\rangle$ and

$$
\varlimsup_{T \rightarrow \infty} \frac{1}{2 T} \int_{-T}^{T}\left|l_{2}(x)\right|^{2} d x=\varlimsup_{T \rightarrow \infty} \frac{1}{T} \int_{0}^{T} f(x) d x=1
$$

where $f$ is defined in Theorem 4.6. By (5.1) and the construction of $f$, we have

$$
\left\|W\left(l_{2}\right)\right\|_{\mathfrak{\gamma}^{2}}^{2}=\varlimsup_{T \rightarrow \infty} \int_{0}^{\infty} 2 f(T x) \frac{\sin ^{2} x}{\pi x^{2}} d x<C_{2}+\varepsilon<1 .
$$

C. $W$ on $\mathfrak{T}^{p}(\mathbf{R})$.

It is well known that for $1 \leqslant p<2$, the Fourier transformation is a contraction from $L^{p}(\mathbf{R})$ into $L^{p^{\prime}}(\mathbf{R}), 1 / p+1 / p^{\prime}=1$. Let $f \in \Re^{p}(\mathbf{R}), 1<p<2$, and let $g=$ $W(f)$. Since $\tau_{h} g-\tau_{-h} g$ is the Fourier transformation of

we have

$$
\sqrt{\frac{2}{\pi}} f(x) \frac{\sin (h x)}{x}
$$

$$
\begin{aligned}
\left(\int_{-\infty}^{\infty} \mid g(u+h)-\right. & \left.\left.g(u-h)\right|^{p^{\prime}} \frac{d u}{\sqrt{2 \pi}}\right)^{1 / p} \\
& \leqslant\left(\int_{-\infty}^{\infty}\left|\sqrt{\frac{2}{\pi}} f(x) \frac{\sin (h x)}{x}\right|^{p} \frac{d x}{\sqrt{2 \pi}}\right)^{1 / p} .
\end{aligned}
$$

This implies

$$
\begin{aligned}
\|g\|_{\gamma_{p}} & =\varlimsup_{h \rightarrow 0^{+}}\left(\frac{1}{2 h} \int_{-\infty}^{\infty}|g(u+h)-g(u-h)|^{p^{\prime}} d u\right)^{1 / p^{\prime}} \\
& \leqslant \varlimsup_{h \rightarrow 0^{+}}\left(\frac{1}{\pi h^{p-1}} \int_{-\infty}^{\infty}\left|f(x) \frac{\sin (h x)}{x}\right|^{p} d x\right)^{1 / p} \\
& =\varlimsup_{T \rightarrow \infty}\left(\int_{-\infty}^{\infty}|f(T x)|^{p}\left|\frac{\sin ^{p} x}{\pi x^{p}}\right| d x\right)^{1 / p}
\end{aligned}
$$

Theorem 5.5. For $1<p<2$, the Wiener transformation $W$ defines a bounded linear operator from $\mathfrak{T}^{p}(\mathbf{R})$ into $\mathcal{T}^{p}(\mathbf{R})$ with

$$
\|W\| \leqslant\left(\int_{0}^{\infty} \tilde{h}(x) d x\right)^{1 / p}
$$

where $h(x)=\left|\left(2 \sin ^{p} x\right) / \pi x^{p}\right|, x>0$.

Proof. The result follows from Theorem 4.5 and (5.3).

\section{REFERENCES}

1. J. Bertrandias, Espaces de fonctions bornés et continues en moyenne asymptotique d'ordre p, Bull. Soc. Math. France 5 (1966).

2. R. Boas, Functions which are odd about several points, Nieuw Arch. Wisk. 1 (1953), 27-32.

3. H. Bohr and E. Fplner, On some types of functional spaces, Acta Math. 76 (1945), 31-155. 
4. F. Carroll, Functions whose differences belong to $L^{P}[0,1]$, Indag. Math. 26 (1964), 250-255.

5. H. Ellis and J. Halperin, Function spaces determined by a levelling length function, Canad. J. Math. 5 (1953), 576-592.

6. G. Hardy and J. Littlewood, Some properties of fractional integrals, Math. Z. 27 (1928), 565-606.

7. E. Hewitt and K. Ross, Abstract harmonic analysis. I, II, Springer-Verlag, Berlin, 1963, 1970.

8. E. Hille and R. Phillips, Functional analysis and semi-groups, Amer. Math. Soc. Colloq. Publ., vol. 31, Amer. Math. Soc., Providence, R.I., 1957.

9. K. Lau, On the Banach spaces of functions with bounded upper means, Pacific J. Math. (to appear).

10. J. Lee, On a class of functions in generalized harmonic analysis, Notices Amer. Math. Soc. 170 (1970), 634. Abstract 674-106.

11. The completeness of the class of functions of bounded upper p-variation, $1<p<\infty$, Notices Amer. Math. Soc. 17 (1970), 1057. Abstract 681-B5.

12. W. Luxemburg and A. Zaanen, Notes on Banach function spaces. I, Indag. Math. 25 (1963), 135-147.

13. J. Marcinkiewicz, Une remarque sur les espaces de M. Besicovitch, C. R. Acad. Sci. Paris 208 (1939), 157-159.

14. P. Masani, On helixes in Banach spaces, Sānkhya 38 (1976), 1-27.

15. An outline of vector graph and conditional Banach spaces, Linear Space and Approximation (P. Butzer and B. Sz.-Nagy, eds.) Birkhäuser-Verlag, Basel, 1978, pp. 72-89.

16. Commentary on the memoire on generalized harmonic analysis [30a], Norbert Wiener: Collected Work, Vol. II, P. Masani, ed. (to appear).

17. R. Nelson, The spaces of functions of finite upper p-variation, Trans. Amer. Math. Soc. 253 (1979), $171-190$.

$\rightarrow$ N. Wiener, Generalized harmonic analysis, Acta Math. 55 (1930), 117-258.

19. _ The Fourier integral and certain of its application, Dover, New York, 1959.

Department of Mathematics, University of Pittsburgh, Pittsburgh, Pennsylvania 15260

U.S. Geological Survey, NSTL Station, MississipPI 39529 